\title{
Organizzazione dell'ambulatorio e del day-hospital nefrologici in un comprensorio di 70.000 abitanti: strutture, strumenti, personale
}

\author{
N. Di Paolo, G. Garosi, A. Guarnieri \\ U.O. Nefrologia e Dialisi USL 30 - Siena
}

e trasformazioni tecnologiche degli utlimi anni hanno messo a disposizione degli operatori sanitari mezzi sempre più efficaci di diagnosi e terapia, inducendo così un aumento della domanda in campo sanitario con dilatazione dei consumi e conseguente lievitazione delle spese. Tali innovazioni hanno apportato un reale beneficio alla collettività nei casi in cui il miglioramento dello stato di salute del singolo individuo si è rivelato definitivo. In altri casi invece (ci riferiamo per esempio ai centri di cobaltoterapia, di terapia intensiva coronarica o di terapia sostitutiva della funzione renale) i risultati ottenuti, pur indiscutibilmente positivi, non si sono tradotti in un guadagno per la società nel senso di garantire al singolo non una guarigione definitiva, ma solo un prolungamento, anche se notevole, della vita. Tutto questo deve essere letto alla luce delle recenti linee di tendenza di politica assistenziale: infatti le risorse di cui unc Stato può disporre non sono in grado di soddisfare tutti i bisogni, per cui appare utile valutare in anticipo ogni intervento sanitario per considerare in che misura un aumento di efficacia dovuto alla introduzione di nuove e costose tecnologie sia tale da giustificarne il prezzo. Da qui lo sviluppo di studi tendenti a valutare la reale convenienza di taluni interventi sanitari, allo scopo di evitare quelli inutili che, sottranedo risorse impiegabili in altre direzioni, risulterebbero in ultima analisi dannosi.

Tra le discipline che hanno beneficiato delle recenti innovazioni tecnologico-sanitarie possiamo senz'altro annoverare quella che si occupa della gestione (intesa come trattamento conservativo o sostitutivo mediante dialisi o trapianto renale) della insufficienza renale terminale. In questo campo si è assistito infatti ad una lievitazione della domanda che riconosce tre cause: il diffondersi della patologia, l'aumento del tasso di sopravvivenza dei pazienti in dialisi conseguente al miglioramento delle tecniche dialitiche ed una minore restrizione nei criteri di selezione dei pazienti da sottomettere a trattamento sostitutivo.

Se si pensa che la popolazione uremica in trattamento nel nostro Paese è passata dalle 4.436 unità del 1974 alle 22.940 del 1988 abbiamo chiaro il peso economico e sanitario assunto da questo fenomeno, del quale troppo spesso trascuriamo l'impatto sociale. La civiltà industriale si poggia sul binomio produzioneconsumo che rappresenta il punto di partenza delle problematiche sociali degli uremici. Spesso la dialisi (in particolar modo quella ospedaliera) costringe il paziente a rinunciare a certi progetti personali e a limitare il proprio impegno nel mondo del lavoro o all'interno della famiglia. Tutto questo può tradursi in motivo di numerose frustrazioni per la perdita del proprio ruolo nella società (inteso come unità produttiva), nella famiglia (all'interno della quale il paziente poteva rappresentare prima della malattia l'elemento portante) e più in generale per la perdita di rapporti di tipo competitivo ed associativo nel tempo libero. Il rapporto con il trattamento sostitutivo è vissuto perciò con profonda ambivalenza esistenziale: da una parte il paziente accetta, per la propria sopravvivenza, la totale dipendenza da esso, dall'altra la rifiuta perché fonte di privazioni e frustrazioni. Ne può derivare un mancato transfert con il personale sanitario, sul quale il paziente tende a scaricare incosciamente le proprie ansie.

Una strategia operativa atta a difendere la comunità dalle patologie uremizzanti $\mathrm{e}$ di conseguenza dagli immensi costi economici e sociali derivanti da esse si fonda essenzialmente su: 1) ricerca epidemiologica, intesa anche come tendenza a valutare la efficacia e la efficienza di un determinato intervento sanitario; 2) campagna di prevenzione delle malattie renali.

\section{Concetti di epidemiologia}

I.'epidemiologia è quella materia che si interessa di come le malattie si distribuiscono nella popolazione, studiandone la nascita, l'andamento, l'eventuale declino, nonché i fattori concausali e comportamentali capaci di favorime l'insorgenza. 
Nei secoli passati fino all'inizio del 1900 l'epidemiologia in generale si è occupata pressoché esclusivamente delle malattie infettive e parassitarie, rappresentando queste le principali cause di mortalità. Tra i primi epidemiologi conosciuti possiamo citare Fracastoro il quale nel suo "De contagione" (1) del 1546 descrive in modo preciso e veramente illuminato le tre modalità di contagio: diretto, semidiretto ed indiretto. Pur non essendo a conoscenza dell'esistenza dei batteri, egli parla di "seminaria" come di particelle invisibili che "si moltiplicano rapidamente e danno origine ai propri simili". Questa materia non ha subito nei secoli grosse innovazioni per vari motivi, primo tra tutti il fatto che le più importanti cause di morte sono rimaste a lungo proprio le malattie infettive e parassitarie; in secondo luogo dobbiamo annoverare la scarsità pressoché assoluta fino al nostro secolo di precise conoscenze etiologiche. Circa 80 anni or sono, con la nascita della moderna microbiologia, l'isolamento dei vari agenti etiologici, l'allestimento di sieri e vaccini e più tardi l'avvento di chemioterapici ed antibiotici, le suddette malattie hanno lasciato il campo ad altre patologie; da qui la necessità da parte degli epidemiologi di studiare queste ultime quanto a modalità di diffusione, fattori favorenti individuali e familiari, influenza di stile di vita ed ambiente di lavoro.

Passando a considerazioni strettamente nefrologiche, possiamo dire che i primi esempi di indagine epidemiologica riguardanti le nefropatie risalgono agli inizi degli anni '60, quando Kaitz e coll. (2) cercarono di trovare nella popolazione una relazione tra batteriuria asintomatica e manifesazioni cliniche dello stato di flogosi delle vie urinarie. Sempre negli anni '60 Ross (3) approfondì lo studio di un certo numero di pazienti microematurici mediante agobiopsia renale, evidenziando un quadro di glomerulonefrite proliferativa focale o diffusa in circa il $70 \%$ dei soggetti esaminati; in seguito Kunin (4) nel 1968 e Tapsall (5) nel 1975 proseguirono gli studi epidemiologici sulla batteriuria asintomatica. Forbes e coll. (6) nel 1969 e Saxena (7) nel 1975 cercarono di documentare invece come le malformazioni delle vie urinarie rappresentassero un fattore favorente l'insorgenza di batteriuria.

In Italia le ricerche epidemiologiche sono iniziate nel 1972 con Scattolini (8) che documentò anomalie urinarie nel $2.67 \%$ dei componenti di una popolazione scolastica; qualche anno più tardi (1974) fu Romagnoli (9) a studiare un insieme di 432 adolescenti mediante esame delle urine e visita medica.

Naturalmente lo studio epidemiologico non deve rimanere fine a se stesso, ma va collegato da una parte alla statistica sanitaria, dall'altra alla educazione sanitaria stessa, costituendo così il primo strumento di lavoro della medicina preventiva. Quest'ultima ha come obbiettivo il miglioramento dello stato di salute della collettività, considerando sia il singolo individuo che la popolazione in gruppo. Tutto ciò può essere realizzato mediante la prevenzione della malattia che si articola in tre punti: prevenzione primaria, secondaria e terziaria.

La prevenzione primaria è tesa all'allontanamento dall'organismo degli agenti morbosi esogeni. Questo tipo di prevenzione si attua in primo luogo a livello ambientale, rimuovendo gli agenti etiologici di malattia e le condizioni che li favoriscono, e secondariamente a livello personale, cercando di individuare i soggetti a rischio prima ancora che si sia instaurata una condizione patologica. Rientra nella prevenzione primaria anche l'istituzione di un servizio di educazione sanitaria. In campo nefrologico è possibile effettuare una fattiva prevenzione primaria con modalità diverse: in primo luogo una collaborazione con Presidi di base ed Istituti di genetica può portare a ridurre la prevalenza di nefropatie congenite ed ereditarie tramite interventi in epoca preconcenzionale; in seconda battuta la collaborazione con Istituti di Medicina del lavoro può coordinare un censimento di tutte le attività a rischio per tale patologia che può rivelarsi l'unico strumento atto a prevenire le malattie professionali; in terzo luogo l'attività delle strutture di base, dei medici di medicina generale e dei farmacisti risulta essenziale per promuovere quella educazione sanitaria indispensabile nel prevenire danni renali da farmaci, da sostanze voluttuarie e da stili di vita inadeguati. Nell'ambito della prevenzione primaria risulta infine essenziale che un Centro nefrologico qualificato promuova un check-up a livello di massa, che a sua volta può essere isolato od avere carattere di periodicità.

La prevenzione secondaria si preoccupa di individuare e quindi allontanare le si- tuazioni iniziali di danno, in cui la malattia esiste, ma è clinicamente asintomatica. In questo stadio di accertamento precoce acquista un notevole interesse una corretta esecuzione dei comuni esami di laboratorio e più in generale delle procedure diagnostiche per 1'apparato urinario, fra le quali l'ecotomografia renale va assumendo una importanza sempre maggiore come esame di primo livello. La prevenzione secondaria si può realizzare principalmente eseguendo uno screening sulla popolazione; tale indagine può essere condotta a livello di massa vagliando a tappeto gli abitanti di una determinata regione, oppure più semplicemente può essere mirata su fasce di popolazione a rischio in funzione di determinati parametri, per esempio presenza di diabete o ipertensione, stato di gravidanza ecc. Tale opera di prevenzione secondaria deve ovviamente fare capo ad un Centro nefrologico che operi in stretta collaborazione con le strutture di base.

La prevenzione terziaria consiste infine nel trattare una situazione morbosa già palese, prevedere eventuali recidive e prevenire l'insorgenza di complicanze; questo è il compito precipuo dei Centri nefrologici.

\section{Epidemiologia della insufficienza renale cronica (IRC)}

Sono passati circa 30 anni da quando il trattamento dialitico, superata la fase sperimentale, ha fatto la propria timida apparizione nei centri clinici d'avanguardia del mondo industrializzato, andando incontro in breve tempo ad una tale diffusione da entrare prepotentemente nella routine di ogni ospedale. Parallelamente si è assistito ad una crescita esponenziale nel numero dei pazienti uremici in trattamento sostitutivo, come dimostrano i dati forniti dall'Associazione Europea di Dialisi e Trapianto: tali rilevazioni, pur risultando relativamente incomplete fino al 1975 , si dimostrano attendibili dopo questa epoca e forniscono nel complesso un quadro abbastanza preciso della evoluzione cui è andata incontro negli ultimi decenni la epidemiologia dell'IRC in trattamento sostitutivo. I dati relativi alla diffusione della IRC in Europa ed in Italia, alla prevalenza degli uremici in Europa e al numero dei Centri di terapia 
sostitutiva in Europa sono riportati nelle Tabelle I-IV.

Da una prima revisione dei dati in nostro possesso appare evidente che il numero degli uremici risulta quadruplicato in Europa in un lasso di tempo pari a 12 anni. Una osservazione più attenta delle tabelle ci suggerisce inoltre alcune considerazioni: 1) l'aumento di prevalenza ed incidenza dell'uremia registrato negli anni '70 non è soltanto l'espressione di una reale diffusione di tale patologia, ma anche di una crescente capacità da parte dei Centri di terapia sostitutiva a soddisfare le richieste di intervento; tale domanda di terapia sostitutiva della funzione renale è stata inoltre sicuramente superiore a quanto risulta dal documentato incremento di pazienti in dialisi; in altre parole l'aumento del numero dei trattamenti sostitutivi effettuati, pur essendo estremamente consistente, non è riuscito a coprire tutta la domanda; 2) le differenze registrate in prevalenza ed incidenza tra le varie aree geografiche stanno ad indicare una minore o maggiore disponibilità economica di alcuni Paesi rispetto ad altri ad attivare Centri per la diagnosi e terapia della IRC: è evidente infatti che lo sviluppo economico di un Paese è direttamente proporzionale alla sua possibilità di fronteggiare determinate patologie con trattamenti particolarmente costosi. Una eccezione a quanto detto è rappresentata dalla Gran Bretagna per i noti criteri di selezione cui sono sottoposti i pazienti affetti da IRC prima di essere ammessi in trattamento sostitutivo; 3 ) l'Italia si colloca per incidenza e prevalenza ben al di sopra della media europea.

Per quanto riguarda la predominanza di sesso nell'IRC, è universalemnte riconosciuto che i maschi risultano molto più colpiti delle femmine (sex ratio 1.5:1); ciò deriva ovviamente da una predominanza maschile sulla incidenza di tutte le cause etiologiche responsabili dell'IRC stessa, eccezion fatta per la nefropatia diabetica, quella da lupus e quella da farmaci (10-15).

Quanto alla distribuzione per età dell'IRC, risulta nella maggior parte dei casi praticamente impossibile risalire alla età di insorgenza dei primi segni di tale patologia e quindi dobbiamo considerare la distribuzione dell'età e l'età media dei pazienti all'ingresso della terapia sostitutiva, come evidenziato nella Tab. V. È interessante notare l'esistenza di
TAB. I - INCIDENZA DELLA INSUFFICIENZA RENALE CRONICA (IRC) NELLA POPOLAZIONE EUROPEA DAL 1975

\begin{tabular}{lccc}
\hline Anno & Incidenza Totale & $\begin{array}{c}\text { Incidenza/milione } \\
\text { di popolazione }\end{array}$ & $\begin{array}{c}\text { Età media dei } \\
\text { nuovi pazienti }\end{array}$ \\
\hline 1975 & 8847 & 16.7 & 43.0 \\
1976 & 9726 & 18.2 & 43.9 \\
1977 & 10116 & 18.7 & 44.8 \\
1978 & 10523 & 21.6 & 46.8 \\
1979 & 12258 & 23.0 & 47.8 \\
1980 & 14080 & 24.6 & \\
1981 & 15889 & 27.7 & \\
1982 & 13508 & 23.5 & \\
1983 & 19668 & 34.3 & \\
1984 & 20815 & 36.3 & \\
1987 & 26161 & 41.4 & \\
1988 & 26960 & 42.3 & \\
\hline
\end{tabular}

TAB. II - PREVALENZA DELLA INSUFFICIENZA RENALE CRONICA (IRC) IN ITALIA DAL 1975

\begin{tabular}{lcc}
\hline Anno & Totale & Casi/milione di abitanti \\
\hline 1975 & 4533 & 81.2 \\
1976 & 5739 & 102.2 \\
1977 & 6440 & 114.1 \\
1978 & 7430 & 131 \\
1979 & 8969 & 158.2 \\
1980 & 11169 & 197 \\
1981 & 13573 & 239 \\
1982 & 12167 & 214.2 \\
1983 & 13021 & 229.2 \\
1984 & 14315 & 252 \\
1987 & 18697 & 327 \\
1988 & 18929 & 330 \\
\hline
\end{tabular}

TAB. III - PREVALENZA DEGLI UREMICI IN EUROPA DAL 1975

\begin{tabular}{lcc}
\hline Anno & Totale & Casi/milione di abitanti \\
\hline 1975 & 27151 & 56.7 \\
1976 & 34215 & 69.1 \\
1977 & 39735 & 73.5 \\
1978 & 44914 & 80.7 \\
1979 & 54550 & 98.9 \\
1980 & 67412 & 117.6 \\
1981 & 80775 & 140.7 \\
1982 & 72164 & 125.7 \\
1983 & 85188 & 146.3 \\
1984 & 89833 & 156.5 \\
1987 & 139732 & 222 \\
1988 & 148103 & 232 \\
\hline
\end{tabular}


TAB. IV - NUMERO DEI CENTRI DI TERAPIA SOSTITUTIVA IN EUROPA DAL 1975

\begin{tabular}{lcc}
\hline Anno & $\begin{array}{c}\text { Centri di terapia } \\
\text { sostitutiva }\end{array}$ & $\begin{array}{c}\text { Centri/milione } \\
\text { di abitanti }\end{array}$ \\
\hline 1975 & 870 & 1.9 \\
1976 & 999 & 2.3 \\
1977 & 1016 & 2.2 \\
1978 & 1090 & 2.3 \\
1979 & 1185 & 2.5 \\
1980 & 1255 & 2.6 \\
1981 & 1344 & 2.8 \\
1982 & 1543 & 2.7 \\
1983 & 1798 & 3.1 \\
1984 & 1904 & 3.3 \\
1987 & 2154 & 3.4 \\
1988 & 2308 & 3.6 \\
\hline
\end{tabular}

TAB. V - DISTRIBUZIONE PERCENTUALE DELL'ETÀ ED ETÀ MEDIA DEI PAZIENTI ALL'INGRESSO IN TERAPIA SOSTITUTIVA IN ALCUNI PAESI ED IN EUROPA NEL 1986

\begin{tabular}{|c|c|c|c|c|c|c|c|c|c|}
\hline \multirow[t]{2}{*}{ Paese } & \multicolumn{8}{|c|}{$\begin{array}{l}\text { Gruppi di età all'ingresso in dialisi } \\
\text { (anni) }\end{array}$} & \multirow{2}{*}{$\begin{array}{l}\text { Età } \\
\text { media } \\
\text { (anni) }\end{array}$} \\
\hline & $0-14$ & $15-24$ & $25-34$ & $35-44$ & $45-54$ & $55-64$ & $65-74$ & $>75$ & \\
\hline Algeria & 2 & 21 & 20 & 19 & 17 & 15 & 6 & 1 & 39 \\
\hline Germania & 1 & 4 & 7 & 10 & 22 & 27 & 21 & 9 & 58 \\
\hline Francia & 3 & 5 & 9 & 11 & 16 & 24 & 20 & 12 & 59 \\
\hline Italia & 2 & 4 & 5 & 10 & 17 & 30 & 23 & 9 & 59 \\
\hline Libia & 0 & 29 & 14 & 33 & 14 & 5 & 5 & 0 & 37 \\
\hline Marocco & 0 & 0 & 4 & 22 & 41 & 26 & 7 & 0 & 51 \\
\hline G. Bret. & 4 & 8 & 10 & 13 & 19 & 26 & 17 & 4 & 53 \\
\hline Tunisia & 1 & 16 & 22 & 19 & 25 & 12 & 4 & 1 & 41 \\
\hline EUROPA & 2 & 6 & 10 & 13 & 19 & 26 & 18 & 6 & 55 \\
\hline
\end{tabular}

una proporzionalità diretta tra età media di ingresso in dialisi e livello socio-economico dei vari Paesi. Questo fatto può essere spiegato con una durata media della vita minore proprio in quei Paesi dove più precoce risulta l'epoca di ingresso in dialisi, oppure con una minore possibilità in atto negli stessi Paesi di diagnosticare precocemente o addirittura prevenire uno stato di IRC; probabilmente però questa correlazione deriva semplicemente dal fatto che in quei Paesi dove le capacità recettive dei Centri di dialisi sono estremamente limitate si rende obbligatoria una accurata selezione dei pazienti da immettere in trattamento sostitutivo. A conferma di quanto detto riportiamo i dati relativi al 1976, quando la recettività dei Centri di dialisi era ben diversa dall'attuale in tutti i Paesi (Tab. VI): a quell'epoca l'età media di ingresso in dialisi era molto più bassa di quella attuale anche nei Paesi più progrediti.

In linea di massima la distribuzione dei vari gruppi sanguigni presso gli uremici in trattamento è apparsa sovrapponibile a quella della popolazione generale, tuttavia in Svizzera e Belgio è stata riscontrata (10) una leggera preponderanza del gruppo $\mathrm{O}$ rispetto al gruppo A, probabilmente legata ad una maggiore frequenza del gruppo $\mathrm{O}$ tra i pazienti affetti da nefropatia da farmaci. L'obesità risulta essere un fattore predisponente allo stato uremico: infatti in tutte le patologie uremizzanti esclusa la nefropatia da farmaci è stato riscontrato (16) che i pazienti sovrappeso iniziano il trattamento dialitico mediamente dieci anni prima rispetto agli altri. Dai dati della letteratura infine non è possibile risalire a considerazioni epidemiologiche valide quanto all'influenza di attività professionale e condizione socio-economica sullo sviluppo di uremia terminale.

Vari Autori (17-25) hanno cercato di stabilire l'incidenza percentuale delle varie patologie renali nel determinismo dell'IRC; è doveroso considerare al riguardo una rilevante difficoltà dovuta al fatto che molti pazienti giungono all'attenzione del nefrologo solo quando dimostrano una IRC in stadio terminale, rendendo in tal modo impossibile una diagnosi etiologica in buona parte dei casi. I dati disponibili in proposito sono riassunti nella Tab. VII. Dalla tabella è facile notare come le glomerulonefriti, la cui epidemiologia verrà discussa dettagliatamente più avanti, rappresentano la causa più frequente di uremia; nel gruppo delle glomerulonefriti appare inoltre notevole il rilievo di una frequenza doppia delle forme primitive rispetto alle secondarie. La diminuzione di prevalenza di queste entità nosologiche, evidenziata invero nelle casistiche più recenti, appare conseguente alla minore tendenza a porre una diagnosi generica di patologia glomerulare quando la nefropatia di base appare di difficile riconoscimento. Sotto la dizione "altre cause" è raggruppato infine anche il danno renale di origine diabetica, la cui incidenza nel determinismo della IRC, in lieve diminuzione negli anni '75-80, ha evidenziato dal 1980 al 1988 un incremento di circa 8 volte $(10,25)$.

Ai fini epidemiologici è interessante notare come il continuo miglioramento delle tecniche di dialisi abbia fatto registrare nel corso del tempo un sensibile aumento della sopravvivenza media dei pazienti in IRC in trattamento sostitutivo. I dati relativi alla sopravvivenza media dei pazienti in dialisi nei periodi 1969-71, 1974-80 e 1982-87 sono riportati in Tab. VIII. Un frettoloso esame di tali dati potrebbe far dedurre che il trend positivo 
TAB. VI - ETÀ MEDIA DI INGRESSO IN DIALISI IN ALCUNI PAESI ED IN EUROPA NEL 1976

\begin{tabular}{lc}
\hline Paese & Età media di ingresso in dialisi \\
Germania & 46.1 \\
Francia & 46.3 \\
Italia & 46.2 \\
Tunisia & 37.9 \\
GB & 37.9 \\
EUROPA & 43.9 \\
\hline
\end{tabular}

TAB. VII - DISTRIBUZIONE PERCENTUALE DELLE CAUSE DI INSUFFICIENZA RENALE CRONICA (IRC)

\begin{tabular}{lcccc}
\hline & $\mathrm{a}$ & $\mathrm{b}$ & $\mathrm{c}$ & $\mathrm{d}$ \\
\hline $\begin{array}{l}\text { Glomerulopatie } \\
\quad \text { primitive } \\
\quad \text { secondarie }\end{array}$ & 38.4 & 32.7 & 32.0 & 24.1 \\
$\begin{array}{l}\text { Nefropatie } \\
\text { interstiziali }\end{array}$ & 23.2 & 23.9 & 21.2 & 10.7 \\
$\begin{array}{l}\text { Nefropatie } \\
\text { vascolari }\end{array}$ & 6.8 & 8.3 & 14.6 & 9.8 \\
$\begin{array}{l}\text { Rene } \\
\text { policistico }\end{array}$ & 8.3 & 9.2 & 9.1 & 8.2 \\
$\begin{array}{l}\text { Nefropatie } \\
\text { ereditarie }\end{array}$ & & & & \\
e congenite & 2.1 & 2.8 & 3.8 & 2.1 \\
Altre cause & 12.4 & 12.7 & 19.3 & 20.2 \\
\begin{tabular}{l} 
Etiologia incerta \\
\hline
\end{tabular} & 8.8 & 10.4 & & 16.4 \\
\hline
\end{tabular}

a: Jacobs e coll. (17)

b: Brunner e coll. (12)

c: Wing e coll. (19)

d: Brunner e coll. (25)

della sopravvivenza risulta meno marcato per i pazienti oltre i 65 anni, ma tale fenomeno è solo apparente: infatti la media della sopravvivenza dei pazienti più anziani nelle epoche più recenti viene drasticamente influenzata in senso negativo dall'abolizione dei criteri selettivi precedentemente in uso per l'ammissione al trattamento sostitutivo. Ciò ha permesso l'ingresso in dialisi anche a quei pazienti (anziani, defedati, cardiopatici e talvolta neoplastici) definiti a rischio che in precedenza venivano esclusi e contri- buisce ad abbassare notevolmente la sopravvienza media della classe ultrasessantacinquenne indipendentemente dal livello qualitativo del trattamento sostitutivo. L'insieme dei dati permette di concludere che l'attesa di vita nelle varie classi di età ha raggiunto livelli accettabili (il 54\% degli uremici con età compresa tra 75 ed 84 anni risulta vivente trend positivo ci autorizza inoltre ad aspettarci negli anni a venire risultati ancora migliori. dopo due anni di trattamento) (25); il

\section{Attività di ambulatorio e day-hospital nefrologico}

La crescita geometrica della popolazione uremica e l'alto costo sociale che la sua gestione comporta impongono il massimo sforzo da parte di un Centro nefrologico nella prevenzione e nella terapia delle malattie renali. È naturale che gli obbiettivi debbano essere necessariamente rivolti a lungo termine. Si dovrà cominciare dalla prevenzione primaria, tesa allo studio epidemiologico e al riconoscimento dei fattori di predisposizione ambientale, familiare e professionale. $\mathrm{Si}$ passerà poi alla prevenzione secondaria, intesa come diagnosi precoce di quelle condizioni di predisposizione individuale allo sviluppo di patologie renali (malformazioni, localizzazione di foci infettivi, diabete mellito). Infine la prevenzione terziaria sarà rivolta al riconoscimento precoce di una malattia renale già in atto, ma ancora affrontabile con interventi che evitino o comunque ritardino la sua progressione.

Senza dubbio, è in buona parte frutto della prevenzione dell'infezione streptococcica il drastico calo della patologia glomerulonefritica dopo l'avvento della terapia antibiotica. Altrettanto importanti, nella prevenzione della IRC, sono stati i successi terapeutici ottenuti nelle nefropatie infettive, vascolari, dismetaboliche, calcolotiche e in uropatie congenite. Un Centro nefrologico deve organizzare programmi di educazione sanitaria da effettuare attraverso le strutture sanitarie di base sui seguenti argomenti:

a) sensibilizzazione delle famiglie sui rischi di nefropatie nei bambini (infezioni, malformazioni congenite, infezioni streptococciche, ecc.);

b) campagne di informazione degli utenti e degli operatori sanitari sull'uso e l'abuso di sostanze potenzialmente nefrotossiche (antibiotici, analgesici, cadmio, ecc.);

c) informazione sulla predisposizione genetica a alcune nefropatie e su malattie con potenziali complicazioni renali (diabete, ipertensione, arteriosclerosi) e misure igienico-sanitarie preventive.

Sono pertanto, a nostro avviso, da incoraggiare campagne epidemiologicopreventive sulla popolazione scolastica, sui lavoratori delle fabbriche e comun- 
que su tutte le comunità del territorio di competenza di un Centro nefrologico.

Attraverso appositi questionari clinicoanamnestici e raccoglitori di campioni delle urine mattutine consegnati ai singoli soggetti e riconsegnati prontamente al Centro, sono possibili screenings seriati di larghi strati di popolazione.

Il Centro nefrologico deve essere attrezzato di un laboratorio atto all'esame a fresco delle urine raccolte, sia attraverso le normali strisce reattive comprensive del Cytur-test, sia attraverso lo studio microscpico del sedimento dei campioni positivi. È anche necessaria la disponibilità di un attrezzato centro di elaborazione che permetta la trascrizione dei dati ricavati dalla scheda e dall'esame delle urine, la loro memorizzazione e la successiva elaborazione.

Le tappe successive di tale indagine epidemiologica saranno quelle di ulteriori accertamenti diagnostici sui soggetti risultati positivi all'accertamento preliminare, da concordare tra il Centro Nefrologico e i medici curanti. Ultimo traguardo sarà quello dell'intervento terapeutico diretto in caso di accertamento di patologia nefrologica e della prevenzione delle complicanze.

È sempre da mettere in evidenza che solo la stretta collaborazione tra tutto il personale operante in un Centro potrà permettere la riuscita dei vari programmi sanitari. I parametri di organizzazione dello staff sanitario devono essere rapportati al variare delle tecnologie e all'aggiungersi di nuove metodiche necessarie al funzionamento ottimale di un Centro. Ciò comporta anche la necessità di differenziare le competenze del personale affidando l'organizzazione, la gestione ed il controllo ad operatori separati per ogni settore, senza tuttavia correre il rischio di creare dei compartimenti stagni, ma dando vita invece ad un tessuto connettivo omogeneo di competenze di livelli operativi che permettano anche, in caso di necessità, interscambio e sostituzione del personale stesso.

È infine da sottolineare l'importanza di coinvolgere nel lavoro altre figure professionali oggi sempre più essenziali per il perfetto funzionamento di un Centro nefrologico (tecnici, assistenti sociali, psicologi, dietisti).

Per quanto riguarda le strutture di degenza, non ci soffermeremo ovviamente sulle caratteristiche di urbanistica ospedaliera che regolano in generale la struttu- razione dei luoghi di degenza. Ci sembra tuttavia opportuno sottolineare l'importanza di dotare le singole stanze di quei requisiti ottimali di igiene e di comfort che dovrebbero caratterizzare l'intera edilizia ospedaliera. Intendiamo riferirci alla riduzione al minimo dei posti letto per ogni stanza, alla dotazione di servizi igienici singoli, alla possibilità di permettere ai ricoverati una qualche vita di relazione in locali dove sia possibile consumare i pasti, leggere, parlare. Nel caso specifico di una degenza nefrologica ci sembra importante la creazione di uno spazio cucina che permetta al personale o al malato in IRC ancora in trattamento dietetico sostitutivo la cottura in loco degli alimenti aproteici: ciò, oltre a rendere più accettabile la dieta da parte del paziente, permette una sicura compliance verso le prescrizioni mediche. Anche il personale operante nel Centro dovrebbe avere a disposizione spazi dove poter discutere i problemi dell'attività di reparto, aggiornare i dati clinico-laboratoristici dei pazienti, studiare. Il problema delle strutture di degenza è ovviamente importantissimo soprattutto per i ricoveri prolungati, ma risulta rilevante anche per l'attività di day-hospital, che dovrebbe usufruire di spazi specificatamente riservati, ma in pratica finisce spesso per condividere gli stessi ambienti delle normali degenze.

La necessità di ottenere spesso in tempi reali valori ematochimici ed urinari di significato diagnostico essenziale (elettroliti, valori emogasanalitici, osmolarità, emocromo ecc. obbliga spesso un Centro nefrologico a dotarsi in proprio delle attrezzature necessarie a tale scopo; questa possibilità risulta particolarmente interessante nell'ambito del day-hospital, in quanto permette di concentrare in una sola mattinata attività diagnostiche e terapeutiche di livello specialistico, con possibilità per il paziente di concentrare in una sola mattinata una notevole mole di accertamenti clinico-diagnostici. La routine diagnostica nefrologica impone poi che l'esame delle urine, ed in particolare il sedimento, venga effettuato in loco ed è pertanto necessario che, in mancanza di tecnici di laboratorio, gli operatori del Centro acquisiscano una competenza tale da rendersi autosufficienti. Necessiterà, quindi, un microscopio a contrasto di fase ed a luce polarizzata. Infine, va ricordato che il sempre maggior uso di farmaci nell'insufficien- za renale ed il monitoraggio nel tempo degli immunosoppressori usati nel trapianto renale stanno sempre più spingendo i Centri di Nefrologia a dotarsi di apparecchiature per il dosaggio di farmaci con tecniche immunoenzimatiche. Il problema dei costi di tali attrezzature è sicuramente rilevante, ma rappresenta l'unico modo per rendere moderno ed efficace un centro operativo.

L'ambulatorio rappresenta a nostro avviso il fulcro essenziale dell'attività di un moderno Centro di Nefrologia; se funziona efficacemente, permette di ridurre quantitativamente e temporalmente $\mathrm{i}$ ricoveri ospedalieri, agendo a vari livelli. In primo luogo l'ambulatorio deve operare come primo filtro specialistico nell'individuazione di nuovi pazienti nefrologici, anche in corso di campagne epidemiologiche, poi come osservatorio del follow-up di pazienti in trattamento farmacologico-dietetico conservativo e come punto di controllo di quei malati in IRC già in trattamento sostitutivo, infine come punto di interscambio con altre strutture sanitarie specialistiche (per patologie quali ipertensione arteriosa, gestosi gravidica, malattie metaboliche, malattie urologiche, litiasi) e come punto di controllo dei pazienti trapiantati. Anche a questo riguardo va detto che le specifiche competenze dei vari operatori sanitari del Centro devono essere valorizzate al massimo con ambulatori separati per gli specifici problemi, ma con la stessa capacità di coordinamneto dell'intera attività ambulatoriale. Un'appendice a nostro avviso assai importante nell'attività di un ambulatorio nefrologico è l'ecografia (26), che permette di avere in tempi brevi, con metodiche non invasive e a basso costo, un valido supporto diagnostico che spesso riesce (insieme al laboratorio intero) a rendere il Centro nefrologico del tutto autonomo nell'attività diagnostica, anche di elevato livello. L'ecotomografia, metodica diagnostica fino a pochi anni fa di competenza esclusivamente radiologica, sta entrando sempre più prepotentemente nell'uso quotidiano della medicina interna in generale e della nefrologia in particolare.

Un Centro di Nefrologia deve essere inoltre in grado di fronteggiare anche attività chirurgiche. Lasciando a Reparti di chirurgia vascolare il compito di eseguire interventi complessi su trochi arteriosi profondi quali superficializzazione e utilizzo di femorali o confezionamento di 
fistole artero-venose in casi complicati, è indubbio che un Centro di Nefrologia debba avere un personale addestrato all'incannulamento di grossi vasi venosi (cateterismo di vena femorale o di succlavia) come accesso di emergenza per pratiche dialitiche. Inoltre, anche le tecniche di dialisi peritoneale richiedono sempre di più al nefrologo di saper posizionare cateteri peritoneali a permanenza: del resto la cosa non sembra essere né impossibile né inaccettabile, visto che la maggior parte delle tecniche di posizionamento di catetere peritoneale sono ideate da nefrologi, che proprio per la loro estrazione internistica hanno sempre ricercato soluzioni semplici ma allo stesso tempo efficaci, alla portata di tutti i loro colleghi. Bisogna infine considerare che ormai la biopsia renale soltanto in rarissimi casi si effettua con tecnica chirurgica essendosi ormai affermato per rapidità, non invasività ed accettabilità del paziente il metodo percutaneo; oggi, inoltre, attraverso la tecnica ecoguidata non esiste praticamente Centro nefrologico che non possa gestire autonomamente un'agobiopsia renale.

E per tutti i motivi elencati che riteniamo necessario nella strutturazioe di un reparto nefrologico la scelta di uno spazio sufficientemente attrezzato da adibire a sala chirurgica con personale responsabile della perfetta disponibilità del materiale necessario in qualsiasi momento del giorno e della notte e per qualsiasi evenienza si renda necessaria.

La rivoluzione informatica è ormai da anni sotto l'occhio di tutti e la medicina in generale e la nefrologia in particolare stanno giorno per giorno ottenendo vantaggi notevoli da essa.

L'introduzione dell'elaborazione dati in un reparto di nefrologia va sempre più estendendosi $(27,28)$ : a partire da un uso puramente "gestionale" di archiviazione dati fino alla possibilità di creare diete rapide e personalizzate per i pazienti nefropatici e di monitorare contemporaneamente l'intera attività di un Centro nefrologico potendo intervenire efficacemente a seconda delle singole necessità.

Assai difficilmente si può sperare che il nefrologo del centro possa essere dall'inizio anche programmatore di computer: può tuttavia diventarlo, anche se ciò comporta l'utilizzo di un'unità solo per tale attività. Ciò che conta è che tra il medico del reparto e il programmatore esista la possibilità di comprendersi, di parlare un linguaggio reciprocamente intellegibile, di porre problemi corretti per una logica informatica che ottengano risposte altrettanto precise. Ma soprattutto è importante che chi opera alla tastiera di un computer sappia dialogare con la macchina, sappia usarne i programmi, possa porre domande e leggere le risposte in modo autonomo.

Anche in questo caso la divisione dei ruoli, convergenti però in un unico programma, risulta essenziale: una volta acquisito il modo di accedere ai programmi chiunque può, -con un minimo di addestramento, operare nell'inserimento dei dati di una campana epidemiologica, nell'uso di programmi non complessi, nella costituzione di un archivio nefrologico ecc.

A nostro avviso, l'uso di un personal computer collegato ad un sistema stampante rappresenta l'unità di base necessaria all'espletamento delle necessità di un Centro. Ad esso si potranno poi via via aggiungere altre attrezzature quali un sistema "plotter" per la scrittura rapida di tabulati, un sistema fotografico autosviluppante collegato col computer per l'elaborazione di documentazione scientifica e di aggiornamento a seconda delle necessità culturali e di produzione scientifica che il Centro sarà in grado di elaborare. Nel caso poi di estese campagne epidemiologiche è chiaro che si dovrà operare un arricchimento della memoria del computer con l'introduzione di programmi sempre più elaborati capaci di effettuare analisi statistico-matematiche complesse o addirittura giungere al collegamento con altre unità nell'ipotesi di lavori multicentrici.

Quello che è importante sottolineare, comunque, è che il computer deve essere al servizio dell'operatore sanitario e non viceversa e che la rigida strutturazione logica della macchina non deve mai e in nessun modo portare ad un analogo modo di pensare e di operare da parte di chi la utilizza.

\section{BIBLIOGRAFIA}

1.

Terson A. Sur Fracastor, son iconographie et les traités de la contagion. Bull Soc Hist Med 1932; 26: 171.

2. Kaitz AL, Williams EJ. Bacteriuria and urinary tract infections in hospitalized patients. N Eng J Med 1960; 262: 425.

3. Ross JH. Recurrent focal nephritis. Am J Med 1960; 391: 406.

4. Kunin CM. Emergence of bacteriuria, proteinuria and simptomatic urinary infections among a population of school girls followed for 7 years. Pediatrics 1968; 41: 968.

5. Tapsall U. Relevance of significant bacteriuria to aetiology and diagnosis of urinary tract infection. Lancet 1975; 2: 637.

6. Forbes PA, Keith ND. Initial urinary tract infection. J Pediatr 1969; 21: 187.

7. Saxena SR. The justification of early $\mathrm{Rx}$ investigation of urinary tract infections in children. Lancet 1975; $2: 403$.

8. Scattolini G. Problemi organizzativi e clinici di un programma di ricerca sistematica delle malattie renali. Atti del IV Corso di aggiornamento di Nefrologia e Metodiche dialitiche, Ospedale Maggiore S. Carlo Borromeo, 29-31 Ottobre 1972, p. 86.

9. Romagnoli D. L'importanza della programmazione sistematica di indagini epidemiologiche atte a svelare precocemente le nefropatie $o$ quei fattori ad esse predisponenti. Atti del I Convegno Regionale Veneto, Venezia 27 Marzo 1986, p. 15.

10. Brynger H, Brunner FP, Chantler W. Combined report on regular dialysis and transpalantation in Europe. Proceedings XVII EDTA, Pitman Medical Publications, 1980. 
11. Broyer M, Brunner FP. Combined report on regular dialysis and transplantation in Europe XII 1981.

12. Brunner FP, Broyer M. Combined report on regular dialysis and transplantation in Europe XV 1984.

13. Jeigler L, Gurland P. Epidemiological and pathofisiological aspects of chronic renal failure. Surgery in chronic renal failure p. 2 .

14. Wing AJ. Why don't the British treat more patients with kidney failure? Br Med J 1987; 287: 1157.

15. Saporiti E. Motivazioni per una indagine clinico statistica sulla dialisi. Giornale veneto di scienze mediche 1983; 4: 35 .

16. Broyer M, Brunner FP. Combined report on regular dialysis and transplantation in Europe XIV 1983.

17. Jacobs C, Brunner FP. Combined report on regular dialysis and transplantation in Europe VII 1976.

18. Dumler F. Renal involvement in type 2 diabetes mellitus; a clinicopathologic study. Kidney Int 1983; 121: 222.

19. Wing AJ, Brunner FP. Combined report on regular dialysis and transpalantation in Europe VIII 1977.

20. Holbing J, Pistor K. Special epidemiological and pathophisiological considerations in children with chronic renal failure. In: Surgery in chronic renal failure, 1986, 8 .

21.

Brynger H, Brunner FP, Chantler W. Combined report on regular dialysis and transplantation in Europe. Proceedings XV EDTA, Pitman Medical Publications, 1978.

22. Royer P, Habib R, Mathieu H, Broyer M. L'insuffisance renal chronique. In: Nephrologie pediatrique, Flammarion Medecine Sciences, $1985 ; 56$.
23. Holliday MA, Potter DA Dobrin RS. Treatment of renal failure in children. Ped Clin North Am 1971; 18: 613.

24. Zillervelo G, Andia J, Gorman HM, Strauss J. Chronic renal failure in children: analysis of main causes and deterioration in 81 children. Int J Ped Nephrol 1980; 1: 30.

25. Brunner FP, Broyer $M$. Combined report on regular dialysis and transplantation in Europe XIX 1988.

26. Di Paolo N, Capotondo L, et al. Validità e limiti dell'ecotomografia renale. Atti del V Simposio della Sezione Tosco-Ligure della Società Italiana di Nefrologia, 1986.

27. Stead W, Garret LE, Hammond WE. Symposium on computing in clinical nephrology. Prectice in nephrology with a computerized medical record. Kidney Intern 1983; 24: 446.

28. Di Giulio S, Degoulet P. L'informatica in nefrologia: alcune esperienze francesi. Atti del IV Convegno della Sez. Regionale Lombarda della Società Italiana di Nefrologia, 1985. 